\section{Análisis de sobrevida en pacientes incidentes de hemodiálisis en Chile, 2013-2019}

\author{
RODRIGO A. SEPÚLVEDA ${ }^{1}$, ANDRÉS PAVLOVIC ${ }^{2}$, \\ OSCAR CORSI ${ }^{3}$, AQUILES JARA ${ }^{1}$
}

\section{Survival analysis of patients starting hemodialysis in Chile between 2013 and 2019}

Background: Renal replacement therapies, especially hemodialysis (HD) in end-stage kidney disease, avoid an inevitable death caused by the disease. However, in elderly patients with multiple comorbidities, this therapy could derive in a comparable survival than conservative management. Considering that HD represents a high cost for the health system, it is worth analyzing the effects of HD on survival. Aim: To analyze the survival and mortality of all national health security system's patients (FONASA) admitted to HD in Chile from 2013 to 2019. Material and Methods: We requested to the Ministry of Health information about all patients affiliated to the public health insurance system that started dialysis between 2013 and 2019. We evaluated the influence of age when starting HD, sex, presence of hypertension, presence of diabetes mellitus (DM), the region of residence, and year of admission on mortality. Results: A total of 24,113 patients aged $61 \pm 15$ years ( $45 \%$ women) were analyzed. Forty five percent of patients were aged $>65$ years. After 5 years of follow-up, the median survival in this age group was 36.1 months. Among patients who started HD at age $>85$ years, the median survival was 14.8 months. Diabetic patients had a median survival of 52.3 months. Advanced age and DM were associated with higher annual mortality. Also, the region of residence and year of admission were associated with higher mortality at 3 and 12 months. Conclusions: The median survival of patients on HD is dependent on age and the presence of comorbidities, among other factors. We performed an analysis to determine if starting $H D$ in older patients with comorbidities has a real benefit over conservative management in terms of survival.

(Rev Med Chile 2020; 148: 1715-1724)

Key words: Chile; Kidney Failure, Chronic; Mortality; Survival; Renal Dialysis.

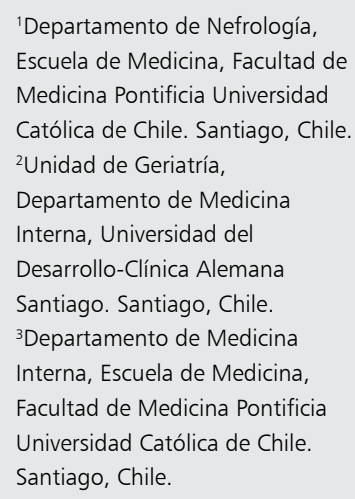

Los autores declaran no tener conflictos de interés.

Trabajo no recibió financiamiento.

Recibido el 12 de agosto de 2020, aceptado el 16 de noviembre de 2020 .

Correspondencia a:

Dr. Rodrigo A. Sepúlveda Departamento de Nefrología. Diagonal Paraguay 362, piso 4.

Santiago, Chile.

rrsepulveda@uc.cl
L a enfermedad renal crónica (ERC) es una patología asociada a mayor morbimortalidad general y cardiovascular, deterioro en la calidad de vida y altos costos para los sistemas de salud $^{1}$. En Chile la última Encuesta Nacional de Salud establece que la prevalencia de filtración glomerular estimada $(\mathrm{eVFG})<60 \mathrm{~mL} / \mathrm{min} / 1,73 \mathrm{~m}^{2}$ es $3 \%$, la cual aumenta hasta $19,1 \%$ en $>65$ años $^{2}$. Sin embargo, esta "definición poblacional de ERC” no incluye todas aquellas alteraciones estructurales, urinarias, glomerulares e hidroelectrolíticas de causa renal que también corresponden a formas de ERC $^{3,4}$.

Debido a la naturaleza progresiva de la ERC 5 , inevitablemente muchos pacientes desarrollarán enfermedad renal crónica terminal (ERCT), momento en el cual es necesario iniciar una terapia de reemplazo renal (TRR). En Chile hay 22.310 
pacientes en Hemodiálisis (septiembre de 2018), 1.285 pacientes en Diálisis Peritoneal (2017) y 7.565 pacientes con un trasplante renal (hasta septiembre 2015$)^{6,7}$.

Las TRR ofrecen una solución a la pérdida de una función orgánica que de otro modo conduciría a la muerte del paciente. Si bien en las últimas décadas ha mejorado la sobrevida de los pacientes en Hemodiálisis (HD), ellos aún presentan un riesgo de mortalidad 10-30 veces mayor al de la población general ${ }^{8}$. En Chile se estima una mortalidad de 10,6 por 100 pacientes-año en pacientes prevalentes en $\mathrm{HD}^{6}$. El mismo indicador en Estados Unidos, países europeos y Japón corresponde aproximadamente a 20, 15 y 6 muertes por 100 pacientes-año respectivamente 9

Este riesgo de mortalidad se concentra en los primeros meses ${ }^{9}$, de hecho, en una cohorte estadounidense, $32 \%$ de todos los fallecimientos durante el primer año ocurría dentro de los 90 días de iniciar $\mathrm{HD}^{10}$.

En nuestro país es escasa la información respecto a la sobrevida de los pacientes incidentes de HD, particularmente durante los primeros meses, aun cuando es una terapia de alto costo y algunas poblaciones podrían tener un beneficio sólo parcial. Por lo anterior, nuestro objetivo es analizar la sobrevida y mortalidad en distintos plazos de los pacientes FONASA incidentes en HD bajo cobertura de las Garantías Explícitas en Salud (GES) en Chile.

\section{Metodología}

Se realizó un estudio de cohorte retrospectivo. La población incluida fueron todos los pacientes que requirieron iniciar terapia de hemodiálisis en Chile con previsión FONASA bajo cobertura GES entre el 1 de enero de 2013 al 31 de diciembre de 2019. Se obtuvo el registro desde los datos del Ministerio de Salud de Chile a través de la Ley de Transparencia, conservando el anonimato de los sujetos. No hubo criterios de exclusión.

Las variables analizadas fueron: edad al iniciar HD, sexo, tiempo de seguimiento, defunción, presencia de Diabetes Mellitus (DM) -tanto tipo 1 como tipo 2- e Hipertensión Arterial (HTA), año de ingreso a HD y región de residencia. Los pacientes provenientes de la Región de Nuble fueron agregados a la Región del Biobío ya que se creó durante el período de estudio. La variable edad, además se dividió en los subgrupos $\leq 65$ años, $>$ 65-75 años, $>75-85$ años $y>85$ años.

Se evaluó la mortalidad y sobrevida de pacientes incidentes en HD durante el período de estudio. Específicamente, se analizaron tasas de mortalidad durante el primer año luego de iniciar HD, como también, la sobrevida a los meses: 1,3 , $6,12,24,36,48$ y 60 posterior al comienzo de HD.

La fecha de inicio de hemodiálisis fue en la que se asignó la garantía de oportunidad en hemodiálisis, ya que a partir de ese momento el paciente debía comenzar HD en menos de 2 semanas. Los pacientes con DM o HTA correspondían a sujetos con estas patologías en los registros de FONASA-GES al momento de iniciar HD.

Para el análisis estadístico se utilizó t de student o $\chi^{2}$ según el caso. Se realizó análisis de sobrevida con curvas de Kaplan-Meier y test de log-rank. Mediante regresión logística múltiple se evaluó la mortalidad a los 3 y 12 meses del inicio de HD, ajustado por: edad al inicio de hemodiálisis, sexo, diagnóstico de HTA y/o DM al iniciar HD, año de inicio de HD y región de residencia.

Se utilizaron los programas Excel ${ }^{\circledR}$, Stata $^{\circledR}$ y GraphPad Prism ${ }^{\circledR}$ para tabulación, análisis y representación de datos. Se aceptó como estadísticamente significativo un valor $\mathrm{p}<0,05$.

El estudio fue aprobado por el Comité Ético-Científico en Ciencias de la Salud de la Facultad de Medicina de la Pontificia Universidad Católica de Chile.

\section{Resultados}

En el período de estudio se analizaron 24.113 pacientes con una media de edad al iniciar HD de 61,07 años $( \pm 14,93)$ con rango entre 0,82 a 98,56 años. De toda esta población $44,5 \%(\mathrm{n}=10.730)$ eran mayores de 65 años y $45,05 \%(n=10.862)$ de sexo femenino. Al iniciar HD 67,45\% $(n=16.264)$ eran hipertensos y $48,81 \%(\mathrm{n}=11.770)$ diabéticos. En la Tabla 1 se representan los datos separados por región. La Figura 1 muestra el número de pacientes que ingresaron a HD cada año durante el período de seguimiento.

Durante todo el período de estudio falleció un $33,54 \%$ de los pacientes $(n=8.088)$. Acumulativamente, de todos los pacientes fallecidos: $4,51 \%$ $(\mathrm{n}=365)$ lo hizo a las 2 semanas de seguimiento; $9,48 \%(\mathrm{n}=767)$ al mes; $33,44 \%(\mathrm{n}=2.705)$ a los 6 meses; $48,01 \%(\mathrm{n}=3.883)$ al año y $95,70 \%$ 
Tabla 1. Características de la población FONASA incidente en HD durante los años 2013 a 2019

\begin{tabular}{|c|c|c|c|c|c|}
\hline Región & $\begin{array}{l}\text { Edad inicio } \\
\text { HD (DE) }\end{array}$ & $\begin{array}{c}\text { Sexo } \\
\text { femenino } \\
(\%)\end{array}$ & $\begin{array}{l}\text { HTA } \\
(\%)\end{array}$ & $\begin{array}{l}\text { DM } \\
(\%)\end{array}$ & $\begin{array}{l}\text { Total pacientes } \\
\text { (\% del total } \\
\text { nacional) }\end{array}$ \\
\hline Arica y Parinacota & $61,00( \pm 14,2)$ & $119(43,3 \%)$ & $165(60,0 \%)$ & $140(50,9 \%)$ & $275 \quad(1,14 \%)$ \\
\hline Tarapacá & $56,59( \pm 15,1)$ & $134(50,6 \%)$ & $166(62,6 \%)$ & $127(47,9 \%)$ & $265(1,10 \%)$ \\
\hline Antofagasta & $61,00( \pm 13,1)$ & $379(43,3 \%)$ & $519(59,3 \%)$ & $440(50,3 \%)$ & $875 \quad(3,63 \%)$ \\
\hline Atacama & $60,74( \pm 14,0)$ & $154(42,1 \%)$ & $206(56,3 \%)$ & $177(48,4 \%)$ & $366(1,52 \%)$ \\
\hline Coquimbo & $60,50( \pm 15,0)$ & $402(45,0 \%)$ & $484(54,1 \%)$ & $381(42,6 \%)$ & $894 \quad(3,71 \%)$ \\
\hline Valparaíso & $60,83( \pm 15,3)$ & $834(44,7 \%)$ & $1.201(64,4 \%)$ & $863(46,3 \%)$ & $1.866(7,74 \%)$ \\
\hline Metropolitana de Santiago & $61,12( \pm 15,1)$ & $4295(44,3 \%)$ & $6.737(69,4 \%)$ & $4.711(48,5 \%)$ & $9.706(40,25 \%)$ \\
\hline $\begin{array}{l}\text { Libertador General Bernardo } \\
\text { O’Higgins }\end{array}$ & $61,95( \pm 15,1)$ & $541(43,6 \%)$ & $892(71,8 \%)$ & $610(49,1 \%)$ & $1.242 \quad(5,15 \%)$ \\
\hline Maule & $60,98( \pm 14,6)$ & $566(43,8 \%)$ & $761(59,0 \%)$ & $586(45,4 \%)$ & $1.291 \quad(5,35 \%)$ \\
\hline Biobío (+Ñuble) & $61,58( \pm 14,9)$ & $1.503(46,1 \%)$ & $2.330(71,4 \%)$ & $1.715(52,6 \%)$ & $3.262(13,53 \%)$ \\
\hline La Araucanía & $61,34( \pm 15,3)$ & $718(48,8 \%)$ & $1.054(71,7 \%)$ & $727(49,5 \%)$ & $1.470 \quad(6,10 \%)$ \\
\hline Los Ríos & $60,20( \pm 14,4)$ & $267(44,8 \%)$ & $407(68,3 \%)$ & $303(50,8 \%)$ & $596 \quad(2,47 \%)$ \\
\hline Los Lagos & $59,61( \pm 14,2)$ & $582(45,5 \%)$ & $891(69,6 \%)$ & $643(50,2 \%)$ & $1.280(5,31 \%)$ \\
\hline $\begin{array}{l}\text { Aysén del General Carlos Ibáñez } \\
\text { del Campo }\end{array}$ & $52,90( \pm 18,2)$ & $35(42,7 \%)$ & $35(42,7 \%)$ & $20(24,4 \%)$ & $82(0,34 \%)$ \\
\hline Magallanes y la Antártica Chilena & $63,64( \pm 14,4)$ & $108(43,7 \%)$ & $148(59,9 \%)$ & $117(47,4 \%)$ & $247 \quad(1,02 \%)$ \\
\hline Desconocida & $64,16( \pm 14,4)$ & $225(56,8 \%)$ & $268(67,7 \%)$ & $210(53,0 \%)$ & $396(1,64 \%)$ \\
\hline Total & $61,07( \pm 14,9)$ & $10.862(45,1 \%)$ & $16.264(67,5 \%)$ & $11.770(48,8 \%)$ & $24.113 \quad(100 \%)$ \\
\hline
\end{tabular}

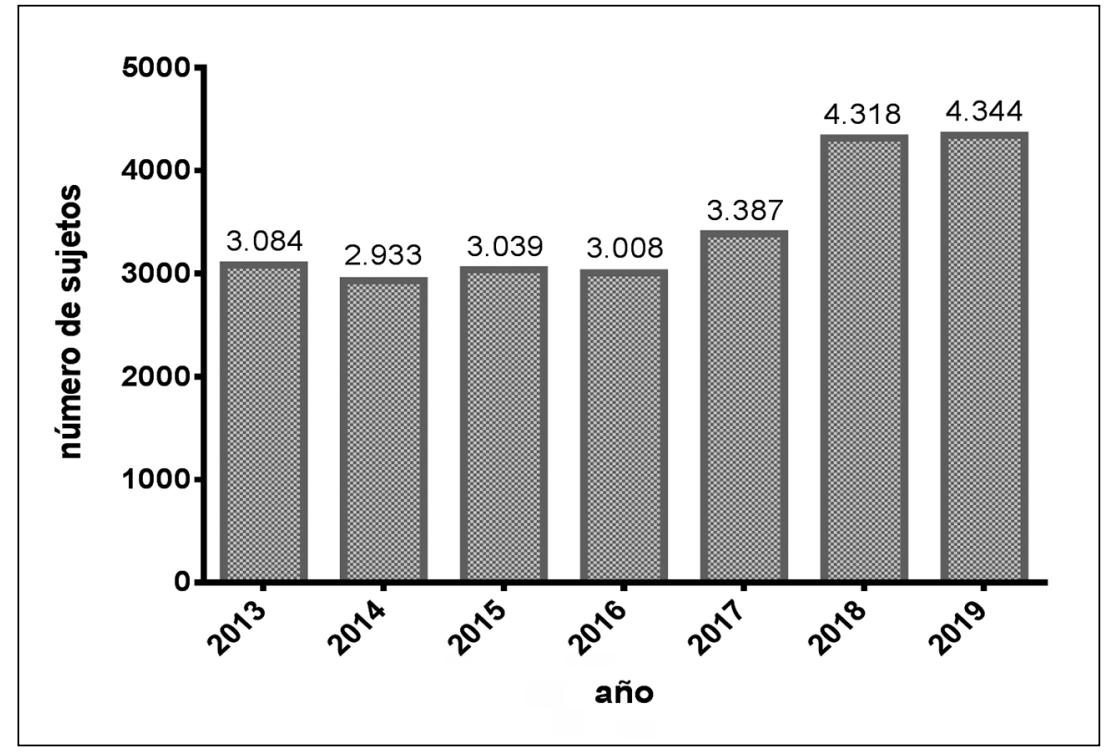

Figura 1. Ingresos anuales a hemodiálisis en el período de seguimiento. 


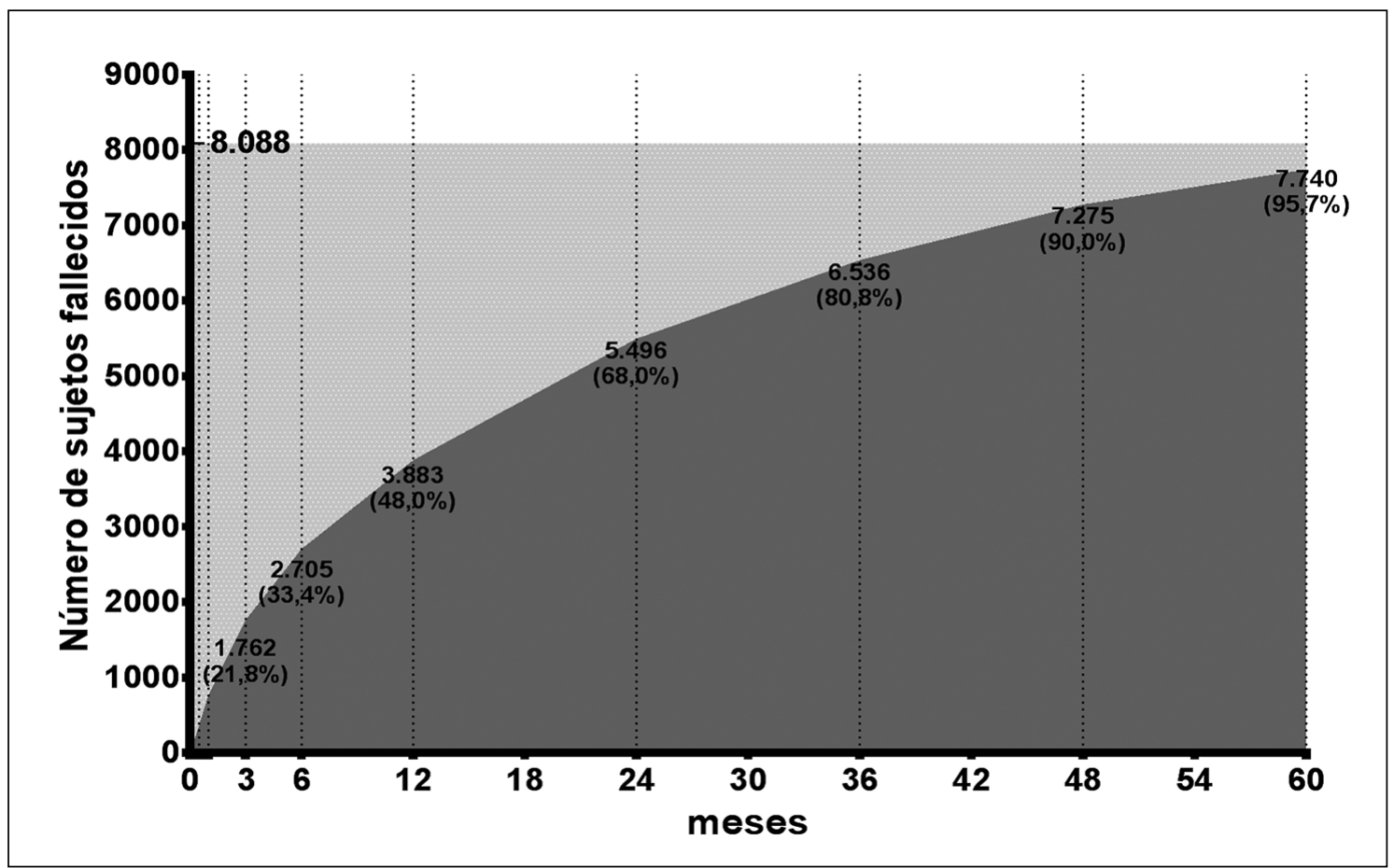

Figura 2. Frecuencia acumulada de pacientes fallecidos en el tiempo respecto del total de fallecidos $(n=8.088)$.

$(\mathrm{n}=7.740)$ a los 5 años (Figura 2).

De todos los pacientes que pudieron tener seguimiento $>3$ meses (es decir, su ingreso a HD ocurrió entre Enero/2013 a Septiembre/2019; $\mathrm{n}=23.033), 7,7 \%(\mathrm{n}=1.763)$ falleció a un plazo de 3 meses (Tabla 2). Específicamente, 11,8\% de los $>65$ años y $4,4 \%$ de los $\leq 65$ fallecieron en los primeros 3 meses de iniciada la HD $(\mathrm{p}<0,001)$.

De los pacientes seguidos por $>1$ año (ingreso a HD entre 2013 y $2018 ; n=19.765), 17,5 \%$ $(\mathrm{n}=3.450)$ falleció a un plazo de 1 año. La edad promedio de los pacientes fallecidos fue 68,37 años $( \pm 12,14)$, mientras que 59,43 años $( \pm 15,01)$ en los que sobrevivieron $(\mathrm{p}<0,001)$. Por grupo etario, al año de seguimiento, 25,6\% de los $>65$ años había fallecido versus $11,0 \%$ en los $\leq 65$ años ( $p$ $<0,001$ ). La Figura 3 muestra las tasas de mortalidad por 100 pacientes-años durante el primer año de seguimiento, con una marcada concentración de mortalidad los primeros 3 meses.

En el análisis multivariado por regresión logística múltiple para mortalidad a los 3 meses de iniciada la $\mathrm{HD}$, se encontró que una mayor edad, año de ingreso a HD y región de residen- cia se asociaron a mayor mortalidad. El sexo y la condición de DM no tuvieron asociación estadística, mientras la presencia de HTA fue un factor protector para mortalidad a 3 meses. El R ${ }^{2}$ ajustado del modelo multivariado fue $6,7 \%$. El mismo modelo logístico para mortalidad a 12 meses demostró que la edad, DM, año de ingreso y región de residencia se asociaron significativamente con mayor mortalidad al año. En cambio, padecer HTA fue un factor protector. Sólo el sexo no tuvo asociación estadística. El $\mathrm{R}^{2}$ ajustado de esta regresión múltiple fue $7 \%$. En la Tabla 3 se muestran los datos de la regresión logística múltiple ajustada para mortalidad anual y la Tabla 4 explicita el análisis por cada región comparado con el resto del país.

En relación a los análisis de sobrevida al año de iniciar HD de los pacientes seguidos por $>1$ año, ésta disminuyó a mayor rango etario (logrank $\mathrm{p}<0,001$ ) (Tabla 2 y Figura 4 ). No hubo diferencias de sobrevida anual según sexo ni HTA $(\log$-rank $\mathrm{p}=0,27 ; \mathrm{p}=0,26$ respectivamente). Sin embargo, quienes eran diabéticos tuvieron menor sobrevida que aquellos que no presentaban esta 
Tabla 2. Datos de sobrevida en pacientes incidentes en hemodiálisis durante 2013 a 2019. Períodos de seguimiento: 1 mes, 3 meses, 6 meses, 1 año, 2 años, 3 años, 4 años y 5 años

\begin{tabular}{|c|c|c|c|c|c|c|c|}
\hline Seguimiento & Condición & $\underset{(\%)^{\dagger}}{n}$ & $\begin{array}{l}\text { Edad } \\
\text { (DE) }\end{array}$ & $\begin{array}{c}\text { Adulto mayor } \\
(\%)^{\ddagger}\end{array}$ & $\begin{array}{c}\text { Sexo femenino } \\
(\%)^{\ddagger}\end{array}$ & $\begin{array}{l}\text { HTA } \\
(\%)^{\ddagger}\end{array}$ & $\begin{array}{l}\text { DM } \\
(\%)^{\ddagger}\end{array}$ \\
\hline \multirow[t]{2}{*}{1 mes } & Vivo & 22.993 & $\begin{array}{c}60,75 \\
( \pm 14,9)^{*}\end{array}$ & $\begin{array}{c}10.017 \\
(45,6 \%)^{*}\end{array}$ & $\begin{array}{l}10.340 \\
(45,0 \%)\end{array}$ & $\begin{array}{c}15.515 \\
(67,5 \%)\end{array}$ & $\begin{array}{l}11.206 \\
(48,7 \%)\end{array}$ \\
\hline & Fallecido & $\begin{array}{c}784 \\
(3,3 \%)\end{array}$ & $\begin{array}{c}70,16 \\
( \pm 11,8)^{*}\end{array}$ & $\begin{array}{c}552 \\
(70,4 \%)^{*}\end{array}$ & $\begin{array}{c}375 \\
(47,8 \%)\end{array}$ & $\begin{array}{c}529 \\
(67,5 \%)\end{array}$ & $\begin{array}{c}393 \\
(50,1 \%)\end{array}$ \\
\hline \multirow[t]{2}{*}{3 meses } & Vivo & 21.270 & $\begin{array}{c}60,35 \\
( \pm 15,0)^{*}\end{array}$ & $\begin{array}{c}9.013 \\
(42,4 \%)^{*}\end{array}$ & $\begin{array}{c}9.540 \\
(44,9 \%)^{*}\end{array}$ & $\begin{array}{c}14.361 \\
(67,5 \%)\end{array}$ & $\begin{array}{l}10.356 \\
(48,7 \%)\end{array}$ \\
\hline & Fallecido & $\begin{array}{c}1.763 \\
(7,7 \%)\end{array}$ & $\begin{array}{c}69,21 \\
( \pm 12,0)^{*}\end{array}$ & $\begin{array}{c}1.203 \\
(68,2 \%)^{*}\end{array}$ & $\begin{array}{c}848 \\
(48,1 \%)^{*}\end{array}$ & $\begin{array}{c}1.199 \\
(68,0 \%)\end{array}$ & $\begin{array}{c}875 \\
(49,6 \%)\end{array}$ \\
\hline \multirow[t]{2}{*}{6 meses } & Vivo & 19.302 & $\begin{array}{c}60,01 \\
( \pm 15,0)^{*}\end{array}$ & $\begin{array}{c}7.991 \\
(41,4 \%)^{*}\end{array}$ & $\begin{array}{c}8.670 \\
(44,9 \%)\end{array}$ & $\begin{array}{c}13.034 \\
(67,5 \%)\end{array}$ & $\begin{array}{c}9.344 \\
(48,4 \%)^{*}\end{array}$ \\
\hline & Fallecido & $\begin{array}{c}2.593 \\
(11,8 \%)\end{array}$ & $\begin{array}{c}68,74 \\
( \pm 11,9)^{*}\end{array}$ & $\begin{array}{c}1.717 \\
(66,2 \%)^{*}\end{array}$ & $\begin{array}{c}1.210 \\
(46,6 \%)\end{array}$ & $\begin{array}{c}1.763 \\
(68,0 \%)\end{array}$ & $\begin{array}{c}1.327 \\
(51,2 \%)^{*}\end{array}$ \\
\hline \multirow[t]{2}{*}{1 año } & Vivo & 16.315 & $\begin{array}{c}59,43 \\
( \pm 15,0)^{*}\end{array}$ & $\begin{array}{c}6.494 \\
(39,8 \%)^{*}\end{array}$ & $\begin{array}{c}7.346 \\
(45,0 \%)\end{array}$ & $\begin{array}{c}11.021 \\
(67,6 \%)\end{array}$ & $\begin{array}{c}7.782 \\
(47,7 \%)^{*}\end{array}$ \\
\hline & Fallecido & $\begin{array}{c}3.450 \\
(17,5 \%)\end{array}$ & $\begin{array}{c}68,4 \\
( \pm 12,1)^{*}\end{array}$ & $\begin{array}{c}2.240 \\
(64,9 \%)^{*}\end{array}$ & $\begin{array}{c}1.588 \\
(46,0 \%)\end{array}$ & $\begin{array}{c}2.367 \\
(68,6 \%)\end{array}$ & $\begin{array}{c}1.819 \\
(52,7 \%)^{*}\end{array}$ \\
\hline \multirow[t]{2}{*}{2 años } & Vivo & 11.267 & $\begin{array}{c}58,34 \\
( \pm 15,1)^{*}\end{array}$ & $\begin{array}{c}4.154 \\
(36,9 \%)^{*}\end{array}$ & $\begin{array}{c}5.055 \\
(44,9 \%)\end{array}$ & $\begin{array}{c}7.561 \\
(67,1 \%)^{*}\end{array}$ & $\begin{array}{c}5.186 \\
(46,0 \%)^{*}\end{array}$ \\
\hline & Fallecido & $\begin{array}{c}4.184 \\
(27,1 \%)\end{array}$ & $\begin{array}{c}67,83 \\
( \pm 12,2)^{*}\end{array}$ & $\begin{array}{c}2.654 \\
(64,4 \%)^{*}\end{array}$ & $\begin{array}{c}1.898 \\
(45,4 \%)\end{array}$ & $\begin{array}{c}2.883 \\
(68,9 \%)^{*}\end{array}$ & $\begin{array}{c}2.287 \\
(54,7 \%)^{*}\end{array}$ \\
\hline \multirow[t]{2}{*}{3 años } & Vivo & 7.809 & $\begin{array}{c}57,42 \\
( \pm 15,2)^{*}\end{array}$ & $\begin{array}{c}2.694 \\
(34,5 \%)^{*}\end{array}$ & $\begin{array}{c}3.522 \\
(45,1 \%)\end{array}$ & $\begin{array}{c}5.233 \\
(67,0 \%)^{*}\end{array}$ & $\begin{array}{c}3.511 \\
(45,0 \%)^{*}\end{array}$ \\
\hline & Fallecido & $\begin{array}{c}4.254 \\
(35,3 \%)\end{array}$ & $\begin{array}{c}67,35 \\
( \pm 12,3)^{*}\end{array}$ & $\begin{array}{c}2.656 \\
(62,4 \%)^{*}\end{array}$ & $\begin{array}{c}1.936 \\
(45,5 \%)\end{array}$ & $\begin{array}{c}2.963 \\
(69,7 \%)^{*}\end{array}$ & $\begin{array}{c}2.369 \\
(55,7 \%)^{*}\end{array}$ \\
\hline \multirow[t]{2}{*}{4 años } & Vivo & 5.250 & $\begin{array}{c}56,27 \\
( \pm 15,3)^{*}\end{array}$ & $\begin{array}{c}1.630 \\
(31,0 \%)^{*}\end{array}$ & $\begin{array}{c}2.382 \\
(45,4 \%)\end{array}$ & $\begin{array}{c}3.464 \\
(66,0 \%)^{*}\end{array}$ & $\begin{array}{c}2.226 \\
(42,4 \%)^{*}\end{array}$ \\
\hline & Fallecido & $\begin{array}{c}3.806 \\
(42,0 \%)\end{array}$ & $\begin{array}{c}66,88 \\
( \pm 12,3)^{*}\end{array}$ & $\begin{array}{c}2.314 \\
(60,8 \%)^{*}\end{array}$ & $\begin{array}{c}1.752 \\
(46,0 \%)\end{array}$ & $\begin{array}{c}2.668 \\
(70,1 \%)^{*}\end{array}$ & $\begin{array}{c}2.155 \\
(56,6 \%)^{*}\end{array}$ \\
\hline \multirow[t]{2}{*}{5 años } & Vivo & 3.128 & $\begin{array}{c}55,02 \\
( \pm 15,2)^{*}\end{array}$ & $\begin{array}{c}871 \\
(27,8 \%)^{*}\end{array}$ & $\begin{array}{c}1.461 \\
(46,7 \%)\end{array}$ & $\begin{array}{c}2.014 \\
(64,4 \%)^{*}\end{array}$ & $\begin{array}{c}1.263 \\
(40,4 \%)^{*}\end{array}$ \\
\hline & Fallecido & $\begin{array}{c}2.885 \\
(48,0 \%)\end{array}$ & $\begin{array}{c}66,75 \\
( \pm 12,4)^{*}\end{array}$ & $\begin{array}{c}1.753 \\
(60,8 \%)^{*}\end{array}$ & $\begin{array}{c}1.335 \\
(46,3 \%)\end{array}$ & $\begin{array}{c}2.013 \\
(69,8 \%)^{*}\end{array}$ & $\begin{array}{c}1.605 \\
(55,6 \%)^{*}\end{array}$ \\
\hline
\end{tabular}

*Diferencia entre grupo vivo vs fallecido con $p<0,05 .{ }^{\dagger}$ Entre paréntesis se muestra porcentaje de fallecidos respecto al total para cada período. *Entre paréntesis se muestra el porcentaje respecto a subgrupos vivo o fallecido para cada período. Se consideran pacientes con oportunidad de seguimiento para cada período. 


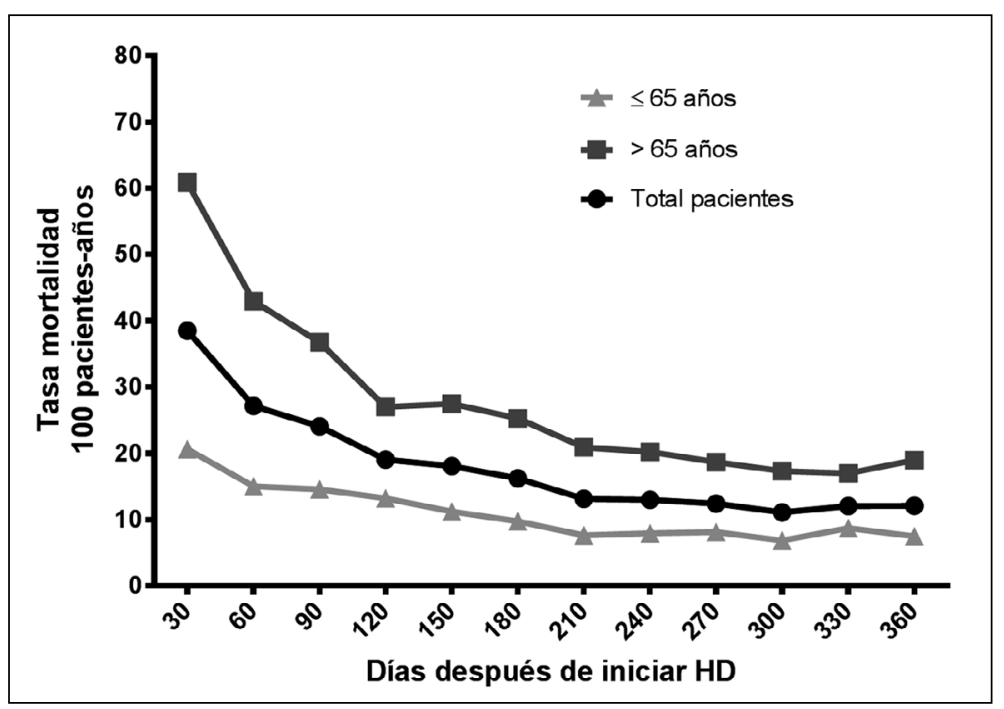

Figura 3. Tasas de mortalidad por pacientes-año los primeros 360 días de iniciar HD.

Tabla 3. Regresión logística múltiple para mortalidad al año de seguimiento ajustado por variables

\begin{tabular}{|lccc|}
\hline & Coeficiente $\boldsymbol{\beta}$ (IC95\%) & Odds Ratio (IC95\%) & Valor p \\
\hline Edad & $0,054(0,051$ a 0,058$)$ & $1,056(1,052$ a 1,060$)$ & $<0,001$ \\
Sexo femenino & $0,001(-0,078$ a 0,080$)$ & $1,001(0,923$ a 1,083$)$ & 0,974 \\
HTA & $-0,402(-0,496$ a $-0,308)$ & $0,669(0,609$ a 0,735$)$ & $<0,001$ \\
DM & $0,136(0,051$ a 0,223$)$ & $1,146(1,052$ a 1,257$)$ & 0,002 \\
Año de ingreso & $-0,058(-0,080$ a $-0,036)$ & $0,944(0,923$ a 0,965$)$ & $<0,001$ \\
Región & $-0,032(-0,043$ a $-0,022)$ & $0,968(0,958$ a 0,978$)$ & $<0,001$ \\
\hline
\end{tabular}

Tabla 4. Análisis de Odds Ratio por región versus el resto del país para mortalidad al año de seguimiento. Regresión logística múltiple ajustada por edad, sexo, HTA, DM y año de ingreso a HD

\begin{tabular}{|lcc|}
\hline Región & Odds Ratio (IC95\%) & Valor p \\
\hline Arica y Parinacota & $1,17(0,83$ a 1,65$)$ & 0,36 \\
\hline Tarapacá & $1,26(0,86$ a 1,84$)$ & 0,23 \\
Antofagasta & $2,19(1,85$ a 2,59$)$ & 0,001 \\
Atacama & $1,16(0,86$ a 1,56$)$ & 0,34 \\
\hline Coquimbo & $0,82(0,66$ a 1,02$)$ & 0,09 \\
Valparaíso & $0,83(0,71$ a 0,97$)$ & 0,02 \\
Metropolitana de Santiago & $0,89(0,82$ a 0,96$)$ & $<0,001$ \\
\hline Libertador General Bernardo O'Higgins & $1,44(1,22$ a 1,69$)$ & $<0,001$ \\
\hline Maule & $1,04(0,87$ a 1,22$)$ & 0,69 \\
\hline Biobío (+Ñuble) & $1,07(0,96$ a 1,19$)$ & 0,24 \\
\hline La Araucanía & $1,04(0,89$ a 1,22$)$ & 0,63 \\
Los Ríos & $0,62(0,47$ a 0,84$)$ & $<0,001$ \\
\hline Los Lagos & $0,69(0,57$ a 0,84$)$ & $<0,001$ \\
\hline Aysén del General Carlos Ibáñez del Campo & $1,25(0,64$ a 2,45$)$ & 0,51 \\
\hline Magallanes y la Antártica Chilena & $0,85(0,59$ a 1,23$)$ & 0,40 \\
\hline
\end{tabular}




\section{Sobrevida según rango etario al año y 5 años de seguimiento}
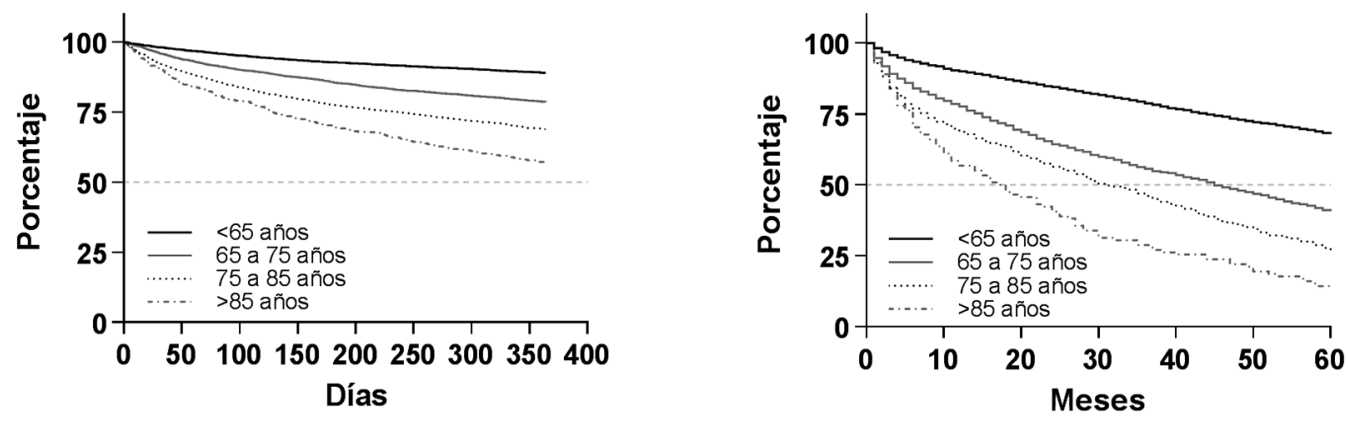

Sobrevida según condición de HTA al año y 5 años de seguimiento
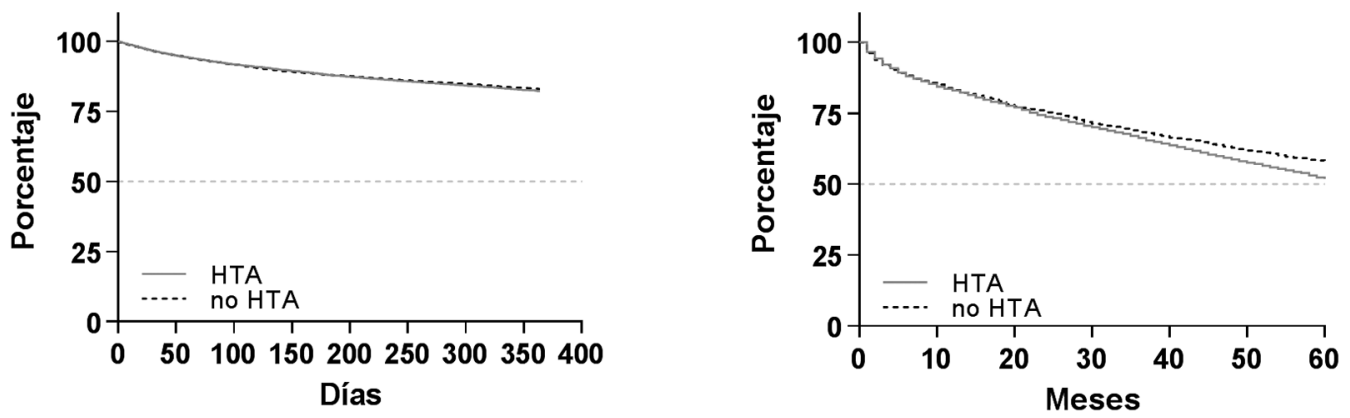

Sobrevida según condición de DM al año y 5 años de seguimiento
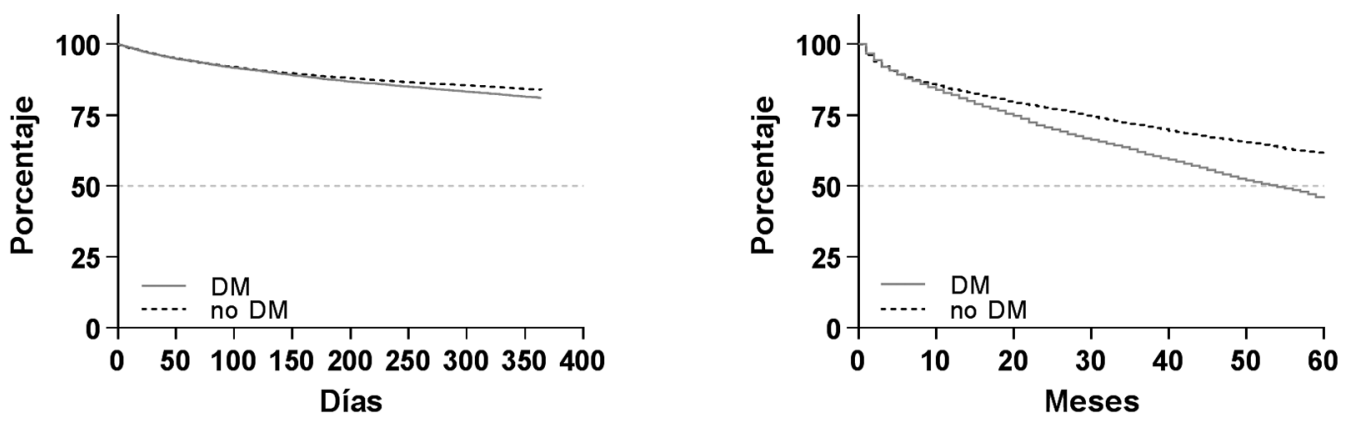

Figura 4. Curvas de sobrevida al año y 5 años de seguimiento.

condición $(\log$-rank p $<0,001)$ (Figura 4).

De todos los pacientes que pudieron ser seguidos por 5 años $(\mathrm{n}=6.013)$, las personas $>65$ años $(\mathrm{n}=2.624)$ tienen menor sobrevida (log-rank $\mathrm{p}<0,001)$ y demuestran una mediana de sobrevida de 36,1 meses. Al contrario, la población $\leq 65$ años $(\mathrm{n}=3.389)$ no presentó mediana de sobrevida, ya que a los 5 años de seguimiento habían fallecido $48,0 \%$ de estos pacientes. Las medianas de sobrevida para los rangos de edad $>65-75$ años, $>75-85$ años $y>85$ años son respectivamente: 44,4 meses, 28,6 meses y 14,8 meses.

A los 5 años de seguimiento, los pacientes que padecen HTA al iniciar HD no logran evidenciar 
mediana de sobrevida, a pesar de que tienen menor sobrevida $(\log$-rank $\mathrm{p}<0,001)$. Los pacientes diabéticos presentan menor sobrevida (log-rank $\mathrm{p}<0,001)$ y una mediana de sobrevida de 52,3 meses (Figura 4). Los adultos mayores que además son diabéticos tienen una mediana de sobrevida de 35,7 meses.

La Tabla 2 resume las características de los pacientes sobrevivientes y fallecidos al mes, 3 meses, 6 meses, 1 año, 2 años, 3 años 4 años y 5 años de seguimiento.

\section{Discusión}

La sobrevida anual de los pacientes incidentes de HD en Chile supera el $80 \%$, comparable con países de mayores ingresos, con una amplia cobertura de 1.260 pacientes por millón de población ${ }^{6}$. No obstante, $7,7 \%$ de los pacientes ha fallecido a los 3 meses de seguimiento; es más, la mortalidad en dicho período fue la más elevada, sobre todo en adultos mayores (Figuras 2 y 3). Esta es una observación que se repite en otros países y que mantiene incertidumbre sobre sus causas ${ }^{9,11}$. Todo esto refuerza nuestra intención de buscar factores que determinan el pronóstico de los pacientes incidentes en HD de tal forma de optimizar su selección y ofrecerles alternativas.

En primer lugar, nuestro trabajo identifica la edad como el factor más importante en la sobrevida. Por ejemplo, la mitad de los pacientes $>85$ años viven más de 14,8 meses, un valor extremadamente alto si consideramos que quienes tienen una eVFG de 6-7 $\mathrm{mL} / \mathrm{min} / 1,73 \mathrm{~m}^{2}$ no viven más de 6 meses $\sin$ TRR $^{12}$. Esto es especialmente relevante teniendo en cuenta el envejecimiento de la población que ingresa a $\mathrm{HD}^{6,13}$. Según los registros de la Sociedad Chilena de Nefrología, el grupo $>61$ años aumentó desde 33,5\% en 1994 hasta 47,2\% en 2018; mientras que el grupo > 81 años aumentó de $1,5 \%$ a $6,2 \%$ en el mismo período ${ }^{6}$.

En Chile, Ríos y cols., analizaron la sobrevida de las personas mayores que iniciaron HD entre 2010 y 2012 en el Servicio de Salud Metropolitano Oriente $^{14}$. Los autores encontraron que la mortalidad fue más alta a mayor edad, alcanzando una mortalidad anual de $43 \%$ en los $>80$ años, mientras que en los $<70$ años, 70-74 años y 75-79 fue de 13, 26 y 29\%, respectivamente. En todos los grupos se observó que el riesgo de muerte era mayor en los primeros meses de iniciada la HD.

Respecto a otros factores, la HTA alcanza una prevalencia de $67,45 \%$ en pacientes incidentes de HD. Este valor es notoriamente mayor a la prevalencia de HTA en la población general ${ }^{2}$, pero inferior a lo reportado en la literatura para pacientes incidentes en $\mathrm{HD}(>80 \%)^{15}$. Lo anterior podría ser secundario a un registro insuficiente del estado de HTA al momento de iniciar HD. Por otro lado, la condición de HTA presenta resultados contradictorios. Mientras en el análisis bivariado se asocia a mayor mortalidad, al realizar el modelo multivariado se comporta como un factor protector. Esto puede explicarse por el efecto de otros factores no ajustados en el análisis bivariado como la edad, o por un efecto protector derivado del acceso a mayor cantidad de controles de salud y así, una eventual mejor adherencia a terapia.

En cuanto a la DM, su análisis debe ser más cauteloso pues incluye a pacientes con DM tipo 1 y DM tipo 2, siendo estos últimos la gran mayoría. La presencia de $\mathrm{DM}$ se asocia a peor pronóstico en pacientes incidentes de HD, sin embargo, la mediana de sobrevida alcanza 52,3 meses, un resultado bastante bueno comparado con lo reportado en otras series ${ }^{16,17}$.

Respecto a indicadores indirectos de calidad, destacamos que los pacientes que ingresaron a HD en años más recientes tienen mejor sobrevida. Esto podría ser consecuencia de distintos factores, por ejemplo, mayor adherencia a las normas de acreditación en los centros de HD o experiencia acumulada por los prestadores. Hubo pacientes con datos de residencia "desconocidos" (1,64\%), quienes además presentaron la mayor mortalidad durante todo el período de seguimiento. Suponemos que estos pacientes provienen de zonas con menor calidad en los registros, e indirectamente, podrían tener menor calidad en su atención de salud. Por otra parte, creemos importante profundizar en las causas de las diferencias en sobrevida entre regiones (documentadas en el análisis multivariado).

En la búsqueda de factores que expliquen los hallazgos de mortalidad se realizó un análisis multivariado. Sin embargo, el modelo sólo explica 7\% del comportamiento de la mortalidad anual de los pacientes incidentes en HD.

Si bien los factores evaluados son de alto impacto en la sobrevida, estos son insatisfactorios para predecir el comportamiento de la mortalidad. 
Así, se puede suponer que existen otras variables que influyen en la sobrevida como factores biológicos, ambientales, sociales y económicos. A modo de ejemplo, un estudio en personas mayores demostró que el aislamiento social tiene un impacto en la sobrevida más importante que la edad o las comorbilidades médicas ${ }^{18}$. Del mismo modo, la fragilidad en pacientes en HD es predictora de disfunción cognitiva, menor calidad de vida $y$ mortalidad ${ }^{19-20}$.

Esto ha motivado la creación de herramientas para la toma de decisiones en el adulto mayor con ERCT que estiman la probabilidad de muerte a 3 y 6 meses luego de iniciar HD, las cuales incorporan casi exclusivamente factores biomédicos ${ }^{22,23}$. No obstante, la utilidad de estas reglas predictivas es limitada. Por ejemplo, una de ellas sólo permite clasificar un grupo de pacientes adultos mayores que presentaría una mortalidad $>40 \%$ a 3 meses $^{22}$, un valor bastante bajo considerando la trascendencia de la decisión que está en juego. En ningún caso la edad por sí sola puede ser el determinante por el cual se rechace la indicación de HD en pacientes mayores.

Por lo tanto, es necesario un enfoque más holístico al decidir el ingreso a HD y no delegarlo en un raciocinio matemático ${ }^{24,25}$. Entonces será fundamental integrar las aspiraciones del paciente, la evaluación del nefrólogo que puede complementarse con un equipo multidisciplinario y las múltiples realidades biológicas, económicas y culturales de la sociedad, el paciente y su familia.

En quienes no ingresen a HD se debería ofrecer cobertura para un manejo conservador, con las medidas paliativas y el acompañamiento que el paciente requiere ${ }^{12}$. Algunos estudios sugieren que en pacientes $>75$ años con múltiples comorbilidades no habría un aumento significativo de la sobrevida al iniciar HD comparado con el manejo conservador $^{26-28}$.

Una limitación de nuestro trabajo es la ausencia de pacientes beneficiarios de sistemas de salud privado, aunque estos representan una mínima proporción de todos los pacientes en HD en Chile, ya que $89,9 \%$ son beneficiarios del sistema de salud público ${ }^{6}$. Por otra parte, desconocemos el tipo de acceso vascular o si los pacientes iniciaron HD durante una hospitalización, lo cual podría asociarse a peor pronóstico. Asimismo, la ausencia de información sobre otras variables de potencial interés, especialmente comorbilidades, restringe el análisis de factores determinantes en la sobrevida. Por desgracia, no tenemos el registro de pacientes que pudieron haber abandonado o suspendido terapia de HD lo que impactaría negativamente en su sobrevida.

Finalmente podemos concluir que en Chile, al igual que el resto del mundo, existe una elevada mortalidad luego de iniciar HD que disminuye en el tiempo. Esta mortalidad inicial no es exhaustivamente predecible. Por otro lado, aquellos que superan este período inicial podrían tener una prolongada sobrevida (similar a la que tienen pacientes en HD de países con altos ingresos). Por lo anterior, la decisión de ingresar a HD no puede basarse sólo en aspectos médicos ni etarios, sino que debe incluir una evaluación integral del enfermo y su entorno.

\section{Referencias}

1. Hill NR, Fatoba ST, Oke JL, Hirst JA, O'Callaghan CA, Lasserson DS, et al. Global Prevalence of Chronic Kidney Disease-A Systematic Review and Meta-Analysis. PLoS One 2016; 11 (7): e0158765.

2. Departamento de Epidemiología, Ministerio de Salud de Chile. Encuesta Nacional de Salud, 2016-2017. Disponible en: epi.minsal.cl/encuesta-nacional-de-salud-2015-2016/ [Consultado el 1 de junio de 2020].

3. International Society of Nephrology. Chapter 1: Definition and classification of CKD. Kidney Int Suppl (2011) 2013; 3 (1): 19-62.

4. Levey AS, Eckardt KU, Tsukamoto Y, Levin A, Coresh J, Rossert J, et al. Definition and classification of chronic kidney disease: a position statement from Kidney Disease: Improving Global Outcomes (KDIGO). Kidney Int 2005; 67 (6): 2089-100.

5. Hommos MS, Glassock RJ, Rule AD. Structural and Functional Changes in Human Kidneys with Healthy Aging. J Am Soc Nephrol 2017; 28 (10): 2838-44.

6. Poblete H. XXXVIII Cuenta de Hemodiálisis Crónica en Chile (al 31 de agosto de 2018). Sociedad Chilena de Nefrología. Disponible en: www.nefro.cl/v2/biblioteca_c. php [Consultado el 11 de junio de 2020].

7. Instituto de Salud Pública, Gobierno de Chile. Registro Nacional de Trasplante 2015. Sociedad Chilena de Nefrología. Disponible en: www.nefro.cl/v2/biblio/ registro/20.pdf [Consultado el 11 de junio de 2020].

8. Msaad R, Essadik R, Mohtadi K, Meftah H, Lebrazi H, Taki H, et al. Predictors of mortality in hemodialysis patients. Pan Afr Med J 2019; 33: 61. 
9. Robinson BM, Zhang J, Morgenstern H, Bradbury BD, $\mathrm{Ng}$ LJ, McCullough KP, et al. Worldwide, mortality risk is high soon after initiation of hemodialysis. Kidney Int 2014; 85 (1): 158-65.

10. Soucie JM, McClellan WM. Early death in dialysis patients: risk factors and impact on incidence and mortality rates. J Am Soc Nephrol 1996; 7 (10): 2169-75.

11. Saran R, Robinson B, Abbott KC, Agodoa LYC, Bragg-Gresham J, Balkrishnan R, et al. US Renal Data System 2018 Annual Data Report: epidemiology of kidney disease in the United States. Am J Kidney Dis 2019; 73 (3) (suppl 1): S1-S772.

12. Murtagh FE, Burns A, Moranne O, Morton RL, Naicker S. Supportive Care: Comprehensive Conservative Care in End-Stage Kidney Disease. Clin J Am Soc Nephrol 2016; 11 (10): 1909-14.

13. Canaud B, Tong L, Tentori F, Akiba T, Karaboyas A, Gillespie B, et al. Clinical practices and outcomes in elderly hemodialysis patients: results from the Dialysis Outcomes and Practice Patterns Study (DOPPS). Clin J Am Soc Nephrol 2011; 6 (7): 1651-62.

14. Ríos Á, Herrera P, Morales Á, Reynolds E, Fernández MB, González F. Análisis de sobrevida en pacientes de edad avanzada que inician hemodiálisis crónica en Servicio de Salud Chileno. Rev Med Chile 2016; 144 (6): 697-703.

15. Zucchelli P, Santoro A, Zuccala A. Genesis and control of hypertension in hemodialysis patients. Semin Nephrol 1988; 8 (2): 163-8.

16. Beladi Mousavi SS, Hayati F, Alemzadeh Ansari MJ, Valavi E, Cheraghian B, Shahbazian H, et al. Survival at 1,3 , and 5 years in diabetic and nondiabetic patients on hemodialysis. Iran J Kidney Dis 2010; 4 (1): 74-7.

17. Vijayan M, Radhakrishnan S, Abraham G, Mathew M, Sampathkumar K, Mancha NP. Diabetic kidney disease patients on hemodialysis: a retrospective survival analysis across different socioeconomic groups. Clin Kidney J 2016; 9 (6): 833-8.

18. Steptoe A, Shankar A, Demakakos P, Wardle J. Social isolation, loneliness, and all-cause mortality in older men and women. Proc Natl Acad Sci USA 2013; 110 (15): 5797-801.

19. McAdams-DeMarco M, Tan J, Salter ML, Gross A, Meoni LA, Jaar BG, et al. Frailty and cognitive function in incident hemodialysis patients. Clin J Am Soc Nephrol 2015; 10 (12): 2181-9.

20. Iyasere O, Brown E, Johansson L, Huson L, Smee J, Maxwell AP, et al. Quality of life and physical function in older patients on dialysis: a comparison of assisted peritoneal dialysis with hemodialysis. Clin J Am Soc Nephrol 2016; 11 (3): 423-30.

21. Johansen KL, Dalrymple LS, Glidden D, Delgado C, Kaysen GA, Grimes B, et al. Association of performance based and self-reported function-based definitions of frailty with mortality among patients receiving hemodialysis. Clin J Am Soc Nephrol 2016; 11 (4): 626-32.

22. Couchoud CG, Beuscart JB, Aldigier JC, Brunet PJ, Moranne OP. Development of a risk stratification algorithm to improve patient-centered care and decision making for incident elderly patients with end-stage renal disease. Kidney Int 2015; 88 (5): 1178-86.

23. Santos J, Oliveira P, Malheiro J, Campos A, Correia S, Cabrita, A et al. Predicting 6-Month Mortality in Incident Elderly Dialysis Patients: A Simple Prognostic Score. Kidney Blood Press Res 2020; 45 (1): 38-50.

24. Koncicki H, Swidler M. Decision making in elderly patients with advanced kidney disease. Clin Geriatr Med 2013; 29 (3): 641-55.

25. Catoni MI, Salas SP, Roessler E, Valdivieso A, Vukusich A, Rivera MS. Decision making among older adults in hemodialysis. A qualitative assessment. Rev Med Chile 2020; 148 (3): 281-7.

26. Carson RC, Juszczak M, Davenport A, Burns A. Is maximum conservative management an equivalent treatment option to dialysis for elderly patients with significant comorbid disease?. Clin J Am Soc Nephrol 2009; 4 (10): 1611-9.

27. Murtagh FE, Marsh JE, Donohoe P, Ekbal NJ, Sheerin NS, Harris FE. Dialysis or not? A comparative survival study of patients over 75 years with chronic kidney disease stage 5. Nephrol Dial Transplant 2007; 22 (7): 1955-62.

28. Chandna SM, Da Silva-Gane M, Marshall C, Warwicker P, Greenwood RN, Farrington K. Survival of elderly patients with stage $5 \mathrm{CKD}$ : comparison of conservative management and renal replacement therapy. Nephrol Dial Transplant 2011; 26 (5): 1608-14. 